\title{
Some new nonlinear integral inequalities and their applications in the qualitative analysis of differential equations
}

\author{
Bin Zheng ${ }^{1 *}$ and Qinghua Feng ${ }^{1,2}$
}

\section{* Correspondence: zhengbin2601@126.com \\ ${ }^{1}$ School of Science, Shandong University of Technology, Zibo, Shandong 255049, China Full list of author information is available at the end of the article}

\begin{abstract}
In this paper, some new nonlinear integral inequalities are established, which provide a handy tool for analyzing the global existence and boundedness of solutions of differential and integral equations. The established results generalize the main results in Sun (J. Math. Anal. Appl. 301, 265-275, 2005), Ferreira and Torres (Appl. Math. Lett. 22, 876-881, 2009), Xu and Sun (Appl. Math. Comput. 182, 1260-1266, 2006) and Li et al. (J. Math. Anal. Appl. 372, 339-349 2010).

MSC 2010: 26D15; 26D10
\end{abstract}

Keywords: integral inequality, global existence, integral equation, differential equation, bounded

\section{Introduction}

During the past decades, with the development of the theory of differential and integral equations, a lot of integral inequalities, for example [1-12], have been discovered, which play an important role in the research of boundedness, global existence, stability of solutions of differential and integral equations.

In [9], the following two theorems for retarded integral inequalities were established.

Theorem A: $R_{+}=[0, \infty)$. Let $u, f, g$ be nondecreasing continuous functions defined on $R_{+}$and let $c$ be a nonnegative constant. Moreover, let $\omega \in C\left(R_{+}, R_{+}\right)$be nondecreasing with $\omega(u)>0$ on $(0, \infty)$ and $\alpha \in C^{1}\left(R_{+}, R_{+}\right)$be nondecreasing with $\alpha(t) \leq t$ on $R_{+}$. $m, n$ are constants, and $m>n>0$. If

$$
u^{m}(t) \leq c^{\frac{m}{m-n}}+\frac{m}{m-n} \int_{0}^{\alpha(t)}\left[f(s) u^{n}(s) \omega(u(s))+g(s) u^{n}(s)\right] d s, \quad t \in R_{+},
$$

then for $t \in[0, \xi]$

$$
u(t) \leq\left\{\Omega^{-1}\left[\Omega\left(c+\int_{0}^{\alpha(t)} g(s) d s\right)+\int_{0}^{\alpha(t)} f(s) d s\right]\right\}^{\frac{1}{m-n}},
$$

where $\Omega(r)=\int_{1}^{r} \frac{1}{\omega\left(s^{\frac{1}{m-n}}\right)} d s, r>0, \Omega^{-1}$ is the inverse of $\Omega, \Omega(\infty)=\infty$, and $\xi \in R_{+}$ is chosen so that $\Omega\left(c+\int_{0}^{\alpha(t)} g(s) d s\right)+\int_{0}^{\alpha(t)} f(s) d s \in \operatorname{Dom}\left(\Omega^{-1}\right)$.

(c) 2011 Zheng and Feng; licensee Springer. This is an Open Access article distributed under the terms of the Creative Commons Attribution License (http://creativecommons.org/licenses/by/2.0), which permits unrestricted use, distribution, and reproduction in any medium, provided the original work is properly cited. 
Theorem B: Under the hypothesis of Theorem B, if

$$
u^{m}(t) \leq c^{\frac{m}{m-n}}+\frac{m}{m-n} \int_{0}^{\alpha(t)} f(s) u^{n}(s) \omega(u(s)) d s+\frac{m}{m-n} \int_{0}^{t} g(s) u^{n}(s) \omega(u(s)) d s, \quad t \in R_{+},
$$

then for $t \in[0, \xi]$

$$
u(t) \leq\left\{\Omega^{-1}\left[\Omega(c)+\int_{0}^{\alpha(t)} f(s) d s+\int_{0}^{t} g(s) d s\right]\right\}^{\frac{1}{m-n}} .
$$

Recently, in [10], the author provided a more general result.

Theorem C: $R_{0}^{+}=[0, \infty), R^{+}=(0, \infty)$. Let $f(t, s)$ and $g(t, s) \in C\left(R_{0}^{+} \times R_{0}^{+}, R_{0}^{+}\right)$be nondecreasing in $t$ for every $s$ fixed. Moreover, let $\phi \in C\left(R_{0}^{+}, R_{0}^{+}\right)$be a strictly increasing function such that $\lim _{x \rightarrow \infty} \phi(x)=\infty$ and suppose that $c \in C\left(R_{0}^{+}, R_{+}\right)$is a nondecreasing function. Further, let $\eta, \omega \in C\left(R_{0}^{+}, R_{0}^{+}\right)$be nondecreasing with $\{\eta, \omega\}(x)>0$ for $x \in(0, \infty)$ and $\int_{x_{0}}^{\infty} \frac{1}{\eta\left(\phi^{-1}(s)\right)} d s=\infty$, with $x_{0}$ defined as below. Finally, assume that $\alpha \in C^{1}\left(R_{0}^{+}, R_{0}^{+}\right)$is nondecreasing with $\alpha(t) \leq t$. If $u \in C\left(R_{0}^{+}, R_{0}^{+}\right)$satisfies

$$
\phi(u(t)) \leq c(t)+\int_{0}^{\alpha(t)}[f(t, s) \eta(u(s)) \omega(u(s))+g(t, s) \eta(u(s))] d s, \quad t \in R_{0}^{+}
$$

then there exists $\tau \in R^{+}$so that for all $t \in[0, \tau]$ we have

$$
\psi(p(t))+\int_{0}^{\alpha(t)} f(t, s) d s \in \operatorname{Dom}\left(\psi^{-1}\right),
$$

and

$$
u(t) \leq \phi^{-1}\left\{G^{-1}\left(\psi^{-1}\left[\psi(p(t))+\int_{0}^{\alpha(t)} f(t, s) d s\right]\right)\right\},
$$

where $G(x)=\int_{x_{0}}^{x} \frac{1}{\eta\left(\phi^{-1}(s)\right)} d s$.

with $x \geq c(0)>x_{0}>0$ if $\int_{0}^{x} \frac{1}{\eta\left(\phi^{-1}(s)\right)} d s=\infty$ and $x \geq c(0)>x_{0} \geq 0$ if

$$
\begin{aligned}
& \int_{0}^{x} \frac{1}{\eta\left(\phi^{-1}(s)\right)} d s<\infty . \\
& p(t)=G(c(t))+\int_{0}^{\alpha(t)} g(t, s) d s \\
& \psi(x)=\int_{x_{1}}^{x} \frac{1}{\omega\left(\phi^{-1}\left(G^{-1}(s)\right)\right)}, x>0, x_{1}>0 .
\end{aligned}
$$

Here $G^{-1}$ and $\psi^{-1}$ are inverse functions of $G$ and $\psi$, respectively.

In [11], $\mathrm{Xu}$ presented the following two theorems:

Theorem $\mathrm{D}: R_{+}=[0, \infty)$. Let $u, f, g$ be real-valued nonnegative continuous functions defined for $x \geq 0, y \geq 0$ and let $c$ be a nonnegative constant. Moreover, let $\omega \in C\left(R_{+}, R_{+}\right)$ be nondecreasing with $\omega(u)>0$ on $(0, \infty)$ and $\alpha, \beta, \in C^{1}\left(R_{+}, R_{+}\right)$be nondecreasing with $\alpha(x) \leq x, \beta(y) \leq y$ on $R_{+} . m, n$ are constants, and $m>n>0$. If

$$
\begin{aligned}
u^{m}(x, y) \leq & a(x)+b(y)+\frac{m}{m-n} \int_{0}^{\alpha(x)} \int_{0}^{\beta(y)}\left[f(t, s) u^{n}(t, s) \omega(u(t, s))\right. \\
& \left.+g(t, s) u^{n}(t, s)\right] d s d t, \quad x, y \in R_{+}
\end{aligned}
$$


then for $x \in[0, \xi], y \in[0, \eta]$

$$
u(x, y) \leq\left\{\Omega^{-1}\left[\Omega(p(x, y))+\int_{0}^{\alpha(x)} \int_{0}^{\beta(y)} f(t, s) d s d t\right]\right\}^{\frac{1}{m-n}}
$$

where

$$
\begin{aligned}
p(x, y)=[ & a(0)+b(y)]^{\frac{m-n}{m}}+\frac{m-n}{m} \int_{0}^{x} \frac{a^{\prime}(t)}{[a(t)+b(0)]^{\frac{n}{m}}} d t \\
& +\int_{0}^{\alpha(x)} \int_{0}^{\beta(y)} g(t, s) d s d t,
\end{aligned}
$$

$\Omega$ is defined as in Theorem A, and $\xi, \eta$ are chosen so that

$$
\Omega(p(x, y))+\int_{0}^{\alpha(x)} \int_{0}^{\beta(y)} f(t, s) d s d t \in \operatorname{Dom}\left(\Omega^{-1}\right) .
$$

Theorem E: Under the hypothesis of Theorem D, if

$$
\begin{aligned}
u^{m}(x, y) \leq & a(x)+b(y)+\frac{m}{m-n} \int_{0}^{\alpha(x)} \int_{0}^{\beta(\gamma)} f(t, s) u^{n}(t, s) \omega(u(t, s)) d s d t \\
& +\frac{m}{m-n} \int_{0}^{x} \int_{0}^{y} g(t, s) u^{n}(t, s) \omega(u(t, s)) d s d t, \quad x, y \in R_{+}
\end{aligned}
$$

then

$$
u(x, y) \leq\left\{\Omega^{-1}\left[\Omega(q(x, y))+\int_{0}^{\alpha(x)} \int_{0}^{\beta(y)} f(t, s) d s d t+\int_{0}^{x} \int_{0}^{y} g(t, s) d s d t\right]\right\}^{\frac{1}{m-n}}
$$

where

$$
q(x, y)=[a(0)+b(y)]^{\frac{m-n}{m}}+\frac{m-n}{m} \int_{0}^{x} \frac{a^{\prime}(t)}{[a(t)+b(0)]^{\frac{n}{m}}} d t .
$$

In this paper, motivated by the above work, we will prove more general theorems and establish some new integral inequalities. Also we will give some examples so as to illustrate the validity of the present integral inequalities.

\section{Main results}

In the rest of the paper we denote the set of real numbers as $R$, and $R_{+}=[0, \infty)$ is a subset of $R$. Dom $(f)$ and $\operatorname{Im}(f)$ denote the definition domain and the image of $f$, respectively.

Theorem 2.1: Assume that $x, a \in C\left(R_{+}, R_{+}\right)$and $a(t)$ is nondecreasing. $f_{i}, g_{i}, h_{i}, \partial_{t} f_{i}$, $\partial_{t} g_{i}, \partial_{t} h_{i} \in C\left(R_{+} \times R_{+}, R_{+}\right), i=1$, 2. Let $\omega \in C\left(R_{+}, R_{+}\right)$be nondecreasing with $\omega(u)>0$ on $(0, \infty) \cdot p, q$ are constants, and $p>q>0$. If $\alpha \in C^{1}\left(R_{+}, R_{+}\right)$is nondecreasing with $\alpha$ $(t) \leq t$ on $R_{+}$, and

$$
\begin{aligned}
x^{p}(t) & \leq a(t)+\int_{0}^{\alpha(t)}\left[f_{1}(s, t) x^{q}(s) \omega(x(s))+g_{1}(s, t) x^{q}(s)+\int_{0}^{s} h_{1}(\tau, t) x^{q}(\tau) d \tau\right] d s \\
& +\int_{0}^{t}\left[f_{2}(s, t) x^{q}(s) \omega(x(s))+g_{2}(s, t) x^{q}(s)+\int_{0}^{s} h_{2}(\tau, t) x^{q}(\tau) d \tau\right] d s, \quad t \in R_{+},
\end{aligned}
$$

then there exists $\bar{t} \in R_{+}$such that for $t \in[0, \bar{t}]$

$$
x(t) \leq\left\{\Omega^{-1}\left\{\Omega(H(t))+\frac{p-q}{p}\left[\int_{0}^{\alpha(t)} f_{1}(s, t)+\int_{0}^{t} f_{2}(s, t) d s\right]\right\}\right\}^{\frac{1}{p-q}},
$$


where $H(t)=a^{\frac{p-q}{p}}(t)+\frac{p-q}{p}\left\{\int_{0}^{\alpha(t)}\left[g_{1}(s, t)+\int_{0}^{s} h_{1}(\tau, t) d \tau\right] d s\right.$

$$
\left.+\int_{0}^{t}\left[g_{2}(s, t)+\int_{0}^{s} h_{2}(\tau, t) d \tau\right] d s\right\}
$$

$\Omega(r)=\int_{1}^{r} \frac{1}{\omega\left(s^{\frac{1}{p-q}}\right)} d s, r>0 . \Omega^{-1}$ is the inverse of $\Omega$, and $\Omega(\infty)=\infty$.

Proof: The proof for the existence of $\bar{t}$ can be referred to Remark 1 in [10]. We notice (3) obviously holds for $t=0$. Now given an arbitrary number $T \in(0, \bar{t}]$, for $t \in$ $(0, T]$, we have

$$
\begin{aligned}
x^{p}(t) \leq & a(T)+\int_{0}^{\alpha(t)}\left[f_{1}(s, t) x^{q}(s) \omega(x(s))+g_{1}(s, t) x^{q}(s)+\int_{0}^{s} h_{1}(\tau, t) x^{q}(\tau) d \tau\right] d s \\
& +\int_{0}^{t}\left[f_{2}(s, t) x^{q}(s) \omega(x(s))+g_{2}(s, t) x^{q}(s)+\int_{0}^{s} h_{2}(\tau, t) x^{q}(\tau) d \tau\right] d s .
\end{aligned}
$$

Let the right-hand side of (4) be $z(t)$, then $x^{p}(t) \leq z(t)$ and $x^{p}(\alpha(t)) \leq z(\alpha(t)) \leq z(t)$. So

$$
\begin{aligned}
z^{\prime}(t)= & {\left[f_{1}(\alpha(t), t) x^{q}(\alpha(t)) \omega(x(\alpha(t)))+g_{1}(\alpha(t), t) x^{q}(\alpha(t))+\int_{0}^{\alpha(t)} h_{1}(\tau, t) x^{q}(\tau) d \tau\right] \alpha^{\prime}(t) } \\
& +\int_{0}^{\alpha(t)}\left[\frac{\partial f_{1}(s, t)}{\partial t} x^{q}(s) \omega(x(s))+\frac{\partial g_{1}(s, t)}{\partial t} x^{q}(s)+\frac{\partial \int_{0}^{s} h_{1}(\tau, t) x^{q}(\tau) d \tau}{\partial t}\right] d s \\
+ & {\left[f_{2}(t, t) x^{q}(t) \omega(x(t))+g_{2}(t, t) x^{q}(t)+\int_{0}^{t} h_{2}(\tau, t) x^{q}(\tau) d \tau\right] } \\
& +\int_{0}^{t}\left[\frac{\partial f_{2}(s, t)}{\partial t} x^{q}(s) \omega(x(s))+\frac{\partial g_{2}(s, t)}{\partial t} x^{q}(s)+\frac{\partial \int_{0}^{s} h_{2}(\tau, t) x^{q}(\tau) d \tau}{\partial t}\right] d s \\
\leq & \left\{\left[f_{1}(\alpha(t), t) \omega(x(\alpha(t)))+g_{1}(\alpha(t), t)+\int_{0}^{\alpha(t)} h_{1}(\tau, t) d \tau\right] \alpha^{\prime}(t)\right. \\
& +\int_{0}^{\alpha(t)}\left[\frac{\partial f_{1}(s, t)}{\partial t} \omega(x(s))+\frac{\partial g_{1}(s, t)}{\partial t}+\frac{\partial \int_{0}^{s} h_{1}(\tau, t) d \tau}{\partial t}\right] d s \\
& +\left[f_{2}(t, t) \omega(x(t))+g_{2}(t, t)+\int_{0}^{t} h_{2}(\tau, t) d \tau\right] \\
& \left.+\int_{0}^{t}\left[\frac{\partial f_{2}(s, t)}{\partial t} \omega(x(s))+\frac{\partial g_{2}(s, t)}{\partial t}+\frac{\partial \int_{0}^{s} h_{2}(\tau, t) d \tau}{\partial t}\right] d s\right\} z^{\frac{q}{p}}(t)
\end{aligned}
$$

Then

$$
\begin{aligned}
\frac{z^{\prime}(t)}{z^{\frac{q}{p}}(t)} \leq & \frac{d \int_{0}^{\alpha(t)}\left[f_{1}(s, t) \omega(x(s))+g_{1}(s, t)+\int_{0}^{s} h_{1}(\tau, t) d \tau\right] d s}{d t} \\
& +\frac{d \int_{0}^{t}\left[f_{2}(s, t) \omega(x(s))+g_{2}(s, t)+\int_{0}^{s} h_{2}(\tau, t) d \tau\right] d s}{d t} .
\end{aligned}
$$

An integration for (5) from 0 to $t$, considering $z(0)=a(T)$, yields

$$
\begin{aligned}
z^{\frac{p-q}{p}}(t) \leq & a^{\frac{p-q}{p}}(T)+\frac{p-q}{p}\left\{\int_{0}^{\alpha(t)}\left[f_{1}(s, t) \omega(x(s))+g_{1}(s, t)+\int_{0}^{s} h_{1}(\tau, t) d \tau\right] d s\right. \\
& \left.+\int_{0}^{t}\left[f_{2}(s, t) \omega(x(s))+g_{2}(s, t)+\int_{0}^{s} h_{2}(\tau, t) d \tau\right] d s\right\} .
\end{aligned}
$$


Then

$$
z^{\frac{p-q}{p}}(t) \leq H(T)+\frac{p-q}{p}\left[\int_{0}^{\alpha(t)} f_{1}(s, t) \omega\left(z^{\frac{1}{p}}(s)\right)+\int_{0}^{t} f_{2}(s, t) \omega\left(z^{\frac{1}{p}}(s)\right) d s\right] .
$$

Let the right-hand side of (7) be $y(t)$. Then we have $z^{\frac{p-q}{p}}(t) \leq y(t)^{\text {, }}$ $z^{\frac{p-q}{p}}(\alpha(t)) \leq \gamma(\alpha(t)) \leq \gamma(t)$, and

$$
\begin{aligned}
y^{\prime}(t)= & \frac{p-q}{p}\left[f_{1}(\alpha(t), t) \omega\left(z^{\frac{1}{p}}(\alpha(t))\right) \alpha^{\prime}(t)+\int_{0}^{\alpha(t)} \frac{\partial f_{1}(s, t)}{\partial t} \omega\left(z^{\frac{1}{p}}(s)\right) d s\right. \\
& \left.+f_{2}(t, t) \omega\left(z^{\frac{1}{p}}(t)\right)+\int_{0}^{t} \frac{\partial f_{2}(s, t)}{\partial t} \omega\left(z^{\frac{1}{p}}(s)\right) d s\right] \\
\leq & \frac{p-q}{p} \frac{d\left[\int_{0}^{\alpha(t)} f_{1}(s, t)+\int_{0}^{t} f_{2}(s, t) d s\right]}{d t} \omega\left(\gamma^{\frac{1}{p-q}}(t)\right),
\end{aligned}
$$

that is

$$
\frac{y^{\prime}(t)}{\omega\left(y^{\frac{1}{p-q}}(t)\right)} \leq \frac{p-q}{p} \frac{d\left[\int_{0}^{\alpha(t)} f_{1}(s, t)+\int_{0}^{t} f_{2}(s, t) d s\right]}{d t}
$$

Integrating (9) from 0 to $t$, considering $y(0)=H(T)$, it follows

$$
\Omega(\gamma(t))-\Omega(H(T)) \leq \frac{p-q}{p}\left[\int_{0}^{\alpha(t)} f_{1}(s, t)+\int_{0}^{t} f_{2}(s, t) d s\right] .
$$

So

$$
\begin{aligned}
& x(t) \leq z^{\frac{1}{p}}(t) \leq y^{\frac{1}{p-q}}(t) \leq \\
& \left\{\Omega^{-1}\left\{\Omega(H(T))+\frac{p-q}{p}\left[\int_{0}^{\alpha(t)} f_{1}(s, t)+\int_{0}^{t} f_{2}(s, t) d s\right]\right\}\right\}^{\frac{1}{p-q}}, t \in(0, \bar{t}] .
\end{aligned}
$$

Taking $t=T$ in (11), then

$$
x(T) \leq\left\{\Omega^{-1}\left\{\Omega(H(T))+\frac{p-q}{p}\left[\int_{0}^{\alpha(T)} f_{1}(s, T)+\int_{0}^{T} f_{2}(s, T) d s\right]\right\}\right\}^{\frac{1}{p-q}},
$$

Considering $T \in(0, \bar{t}]$ is arbitrary, substituting $T$ with $t$, and then the proof is complete.

Remark 1 : We note that the right-hand side of (2) is well defined since $\Omega(\infty)=\infty$.

Remark 2 : If we take $p=2, q=1, \omega(u)=u, h_{1}(s, t)=h_{2}(s, t) \equiv 0$ or $p=2, q=1, h_{1}(s$, $t)=h_{2}(s, t) \equiv 0$, respectively, then our Theorem 2.1 reduces to [12, Theorems 2.1, 2.2].

Corollary 2.1: Assume that $x, a, \alpha, \omega, \Omega$ are defined as in Theorem 2.1. $f_{i}, g_{i}, h_{i} \in C$ $\left(R_{+}, R_{+}\right), m_{i}, n_{i}, l_{i} \in C^{1}\left(R_{+}, R_{+}\right), i=1,2$. If

$$
\begin{aligned}
x^{p}(t) & \leq a(t)+\int_{0}^{\alpha(t)}\left[m_{1}(t) f(s) x^{q}(s) \omega(x(s))+n_{1}(t) g_{1}(s) x^{q}(s)\right. \\
& \left.+\int_{0}^{s} l_{1}(t) h_{1}(\tau) x^{q}(\tau) d \tau\right] d s+\int_{0}^{t}\left[m_{2}(t) f_{2}(s) x^{q}(s) \omega(x(s))+n_{2}(t) g_{2}(s) x^{q}(s)\right. \\
& \left.+\int_{0}^{s} l_{2}(t) h_{2}(\tau) x^{q}(\tau) d \tau\right] d s, \quad t \in R_{+}
\end{aligned}
$$


then we can find some $\bar{t} \in R_{+}$such that for $t \in[0, \bar{t}]$

$$
x(t) \leq\left\{\Omega^{-1}\left\{\Omega(H(t))+\frac{p-q}{p}\left[\int_{0}^{\alpha(t)} m_{1}(t) f_{1}(s) d s+\int_{0}^{t} m_{2}(t) f_{2}(s) d s\right]\right\}\right\}^{\frac{1}{p-q}},
$$

where

$$
\begin{aligned}
H(t)= & a^{\frac{p-q}{p}}(t)+\frac{p-q}{p}\left\{\int_{0}^{\alpha(t)}\left[n_{1}(t) g_{1}(s)+\int_{0}^{s} l_{1}(t) h_{1}(\tau) d \tau\right] d s\right. \\
& \left.+\int_{0}^{t}\left[n_{2}(t) g_{2}(s)+\int_{0}^{s} l_{2}(t) h_{2}(\tau) d \tau\right] d s\right\} .
\end{aligned}
$$

Remark 3: If $a(t) \equiv C^{\frac{p}{p-q}}, m_{1}(t)=n_{1}(t) \equiv 1, l_{1}(t) \equiv 0, m_{2}(t)=n_{2}(t)=l_{2}(t) \equiv 0$ for $t \in$ $R^{+}$, then Corollary 1 reduces to Theorem A [9, Theorem 2.1]. If $a(t) \equiv C^{\frac{p}{p-q}}, m_{1}(t) \equiv$ $1, g_{1}(t) \equiv 0, l_{1}(t) \equiv 0, m_{2}(t) \equiv 1, n_{2}(t)=l_{2}(t) \equiv 0$ for $t \in R^{+}$, then Corollary 2.1 reduces to Theorem B [9, Theorem 2.2].

Corollary 2:2: Assume that $x, a, \alpha, \omega, \Omega$ are defined as in Theorem 2.1. $f, g, h, \partial_{t} f$, $\partial_{t} g, \partial_{t} h \in C\left(R_{+} \times R_{+}, R_{+}\right)$. If

$$
x^{p}(t) \leq a(t)+\int_{0}^{\alpha(t)}\left[f(s, t) x^{q}(s) \omega(x(s))+g(s, t) x^{q}(s)+\int_{0}^{s} h(\tau, t) x^{q}(\tau) d \tau\right] d s, t \in R_{+},
$$

then for $t \in[0, \bar{t}]$

$$
x(t) \leq\left\{\Omega^{-1}\left[\Omega(H(t))+\frac{p-q}{p} \int_{0}^{\alpha(t)} f(s, t) d s\right]\right\}^{\frac{1}{p-q}},
$$

where

$$
H(t)=a^{\frac{p-q}{p}}(t)+\frac{p-q}{p}\left\{\int_{0}^{\alpha(t)}\left[g(s, t)+\int_{0}^{s} h(\tau, t) d \tau\right] d s\right\} .
$$

Corollary 2:3: Assume that $x, a, \alpha, \omega, \Omega$ are defined as in Theorem 2.1. $f, g, h \in C(R$ $\left.{ }_{+}, R_{+}\right), m, n, l \in C^{1}\left(R_{+}, R_{+}\right)$. If

$$
\begin{aligned}
x^{p}(t) \leq & a(t)+\int_{0}^{\alpha(t)}\left[m(t) f(s) x^{q}(s) \omega(x(s))+n(t) g(s) x^{q}(s)\right. \\
& \left.+\int_{0}^{s} l(t) h(\tau) x^{q}(\tau) d \tau\right] d s, t \in R_{+},
\end{aligned}
$$

then for $t \in[0, \bar{t}]$

$$
x(t) \leq\left\{\Omega^{-1}\left[\Omega(H(t))+\frac{p-q}{p} \int_{0}^{\alpha(t)} m(t) f(s) d s\right]\right\}^{\frac{1}{p-q}},
$$

where

$$
H(t)=a^{\frac{p-q}{p}}(t)+\frac{p-q}{p}\left\{\int_{0}^{\alpha(t)}\left[n(t) g(s)+\int_{0}^{s} l(t) h(\tau) d \tau\right] d s\right\} .
$$

Motivated by Corollary 2.2 and Theorem C [10], we will give the following more general theorem:

Theorem 2:2: Assume that $f(s, t), g(s, t), h(s, t) \in C\left(R_{+} \times R_{+}, R_{+}\right)$are nondecreasing in $t$ for each $s$ fixed, and $\varphi \in C\left(R_{+}, R_{+}\right)$is a strictly increasing function with 
$\lim _{x \rightarrow \infty} \phi(x)=\infty . \psi, \omega \in C\left(R_{+}, R_{+}\right)$are nondecreasing with $\psi(x)>0, \omega(x)>0$ for $x \in$ $(0, \infty)$ and $\int_{t_{0}}^{\infty} \frac{1}{\psi\left(\phi^{-1}(s)\right)} d s=\infty, a(t), \alpha(t)$ are defined as in Theorem 2.1, and $a(0)>$ $t_{0}>0$. If $x \in C\left(R_{+}, R_{+}\right)$satisfies the following integral inequality containing multiple integrals

$$
\begin{aligned}
\phi(x(t)) \leq a(t) & +\int_{0}^{\alpha_{1}(t)} f(s, t) \psi(x(s)) d s+\int_{0}^{\alpha_{2}(t)} g(s, t) \psi(x(s)) \omega(x(s)) d s \\
& +\int_{0}^{\alpha_{3}(t)} \int_{0}^{s} h(\tau, t) \psi(x(\tau)) d \tau d s,
\end{aligned}
$$

then we can find some $\bar{t} \in R_{+}$such that for $t \in[0, \bar{t}]$

$$
Y(\bar{H}(t))+\int_{0}^{\alpha_{2}(t)} g(s, t) d s \in \operatorname{Dom}\left(Y^{-1}\right),
$$

and

$$
x(t) \leq \phi^{-1}\left\{J^{-1}\left[Y^{-1}\left(Y(\bar{H}(t))+\int_{0}^{\alpha_{2}(t)} g(s, t) d s\right)\right]\right\},
$$

where

$$
\begin{aligned}
& \bar{H}(t)=J(a(t))+\int_{0}^{\alpha_{1}(t)} f(s, t) d s+\int_{0}^{\alpha_{3}(t)} \int_{0}^{s} h(\tau, t) d \tau d s \\
& J(t)=\int_{t_{0}}^{t} \frac{1}{\psi\left(\phi^{-1}(s)\right)} d s, t>t_{0}, Y(t)=\int_{t_{1}}^{t} \frac{1}{\omega\left(\phi^{-1}\left(J^{-1}(s)\right)\right)} d s, t_{1}>0, t>0 .
\end{aligned}
$$

Proof: The proof for the existence of $\bar{t}$ can be referred to Remark 1 in [10]. We notice (22) obviously holds for $t=0$. Now given an arbitrary number $T>0, T \in(0, \bar{t}]$. Define

$$
\begin{aligned}
d(t)= & a(T)+\int_{0}^{\alpha_{1}(t)} f(s, T) \psi(x(s)) d s+\int_{0}^{\alpha_{2}(t)} g(s, T) \psi(x(s)) \omega(x(s)) d s \\
& +\int_{0}^{\alpha_{3}(t)} \int_{0}^{s} h(\tau, T) \psi(x(\tau)) d \tau d s .
\end{aligned}
$$

Then for $t \in(0, T]$,

$$
x(t) \leq \phi^{-1}(d(t))
$$

and

$$
\begin{aligned}
d^{\prime}(t)= & f\left(\alpha_{1}(t), T\right) \psi\left(x\left(\alpha_{1}(t)\right)\right) \alpha^{\prime}{ }_{1}(t)+g\left(\alpha_{2}(t), T\right) \psi\left(x\left(\alpha_{2}(t)\right)\right) \omega\left(x\left(\alpha_{2}(t)\right)\right) \alpha^{\prime}{ }_{2}(t) \\
& +\alpha^{\prime}{ }_{3}(t) \int_{0}^{\alpha_{3}(t)} h(\tau, T) \psi(x(\tau)) d \tau \\
\leq & f\left(\alpha_{1}(t), T\right) \psi\left(\phi^{-1}\left(d\left(\alpha_{1}(t)\right)\right)\right) \alpha^{\prime}{ }_{1}(t)+g\left(\alpha_{2}(t), T\right) \psi\left(\phi^{-1}\left(d\left(\alpha_{2}(t)\right)\right)\right) \\
& \omega\left(\phi^{-1}\left(d\left(\alpha_{2}(t)\right)\right)\right) \alpha^{\prime}{ }_{2}(t)+\alpha^{\prime}{ }_{3}(t) \psi\left(\phi^{-1}\left(d\left(\alpha_{3}(t)\right)\right)\right) \int_{0}^{\alpha_{3}(t)} h(\tau, T) d \tau \\
\leq & f\left(\alpha_{1}(t), T\right) \psi\left(\phi^{-1}(d(t))\right) \alpha^{\prime}{ }_{1}(t)+g\left(\alpha_{2}(t), T\right) \psi\left(\phi^{-1}(d(t))\right) \\
& \omega\left(\phi^{-1}\left(d\left(\alpha_{2}(t)\right)\right)\right) \alpha^{\prime}{ }_{2}(t)+\alpha^{\prime}{ }_{3}(t) \psi\left(\phi^{-1}(d(t))\right) \int_{0}^{\alpha_{3}(t)} h(\tau, T) d \tau .
\end{aligned}
$$


So

$$
\begin{aligned}
\frac{d^{\prime}(t)}{\psi\left(\phi^{-1}(d(t))\right)} \leq & f\left(\alpha_{1}(t), T\right) \alpha^{\prime}{ }_{1}(t)+g\left(\alpha_{2}(t), T\right) \omega\left(\phi^{-1}\left(d\left(\alpha_{2}(t)\right)\right)\right) \alpha^{\prime}{ }_{2}(t) \\
& +\alpha^{\prime}{ }_{3}(t) \int_{0}^{\alpha_{3}(t)} h(\tau, T) d \tau .
\end{aligned}
$$

Integrating (27) from 0 to $t$, considering $J$ is increasing, we can obtain

$$
\begin{aligned}
d(t) \leq & J^{-1}\left[J(a(T))+\int_{0}^{\alpha_{1}(t)} f(s, T) d s+\int_{0}^{\alpha_{2}(t)} g(s, T) \omega\left(\phi^{-1}(d(s))\right) d s\right. \\
& \left.+\int_{0}^{\alpha_{3}(t)} \int_{0}^{s} h(\tau, T) d \tau d s\right] \\
\leq & J^{-1}\left[\bar{H}(T)+\int_{0}^{\alpha_{2}(t)} g(s, T) \omega\left(\phi^{-1}(d(s))\right) d s\right], \quad t \in(0, T] .
\end{aligned}
$$

Define $G(t)=\bar{H}(T)+\int_{0}^{\alpha_{2}(t)} g(s, T) \omega\left(\phi^{-1}(d(s))\right) d s$, then

$$
d(t) \leq J^{-1}(G(t)), \quad t \in(0, T],
$$

and

$$
\begin{aligned}
G^{\prime}(t) & =g\left(\alpha_{2}(t), T\right) \omega\left(\phi^{-1}\left(d\left(\alpha_{2}(t)\right)\right)\right) \alpha^{\prime}{ }_{2}(t) \\
& \leq g\left(\alpha_{2}(t), T\right) \omega\left(\phi^{-1}\left(J^{-1}\left(G\left(\alpha_{2}(t)\right)\right)\right)\right) \alpha^{\prime}{ }_{2}(t) \\
& \leq g\left(\alpha_{2}(t), T\right) \omega\left(\phi^{-1}\left(J^{-1}(G(t))\right) \alpha^{\prime}{ }_{2}(t),\right.
\end{aligned}
$$

that is,

$$
\frac{G^{\prime}(t)}{\omega\left(\phi^{-1}\left(J^{-1}(G(t))\right)\right.} \leq g\left(\alpha_{2}(t), T\right) \alpha_{2}^{\prime}(t) .
$$

Integrating (31) from 0 to $t$, considering $G(0)=\bar{H}(T)$ and $Y$ is increasing, it follows

$$
G(t) \leq Y^{-1}\left[Y(\bar{H}(T))+\int_{0}^{\alpha_{2}(t)} g(s, T) d s\right], \quad t \in(0, T] .
$$

Combining (25), (29) and (32) we have

$$
x(t) \leq \phi^{-1}\left\{J^{-1}\left[Y^{-1}\left(Y(\bar{H}(T))+\int_{0}^{\alpha_{2}(t)} g(s, T) d s\right)\right]\right\}, \quad t \in(0, T] .
$$

Taking $t=T$ in (33), it follows

$$
x(T) \leq \phi^{-1}\left\{J^{-1}\left[Y^{-1}\left(Y(\bar{H}(T))+\int_{0}^{\alpha_{2}(T)} g(s, T) d s\right)\right]\right\} .
$$

Considering $T \in(0, \bar{t}]$ is arbitrary, substituting $T$ with $t$ we have completed the proof.

Remark 4: If $h(s, t) \equiv 0, \alpha_{1}(t)=\alpha_{2}(t)=\alpha(t)$, then Theorem 2.2 becomes Theorem C [10, Theorem 1].

Now we will apply the concept of establishing Theorem 2.2 to the situation with two independent variables.

Theorem 2:3: Assume that $f_{i}(x, y), g_{i}(x, y), h_{i}(x, y) \in C\left(R_{+} \times R_{+}, R_{+}\right), i=1,2$, and $\varphi \in C$ $\left(R_{+}, R_{+}\right)$is a strictly increasing function with $\lim _{x \rightarrow \infty} \phi(x)=\infty . a(x, y) \in C\left(R_{+} \times R_{+}, R_{+}\right)$is 
nondecreasing in $x$ for every fixed $y$ and nondecreasing in $y$ for every fixed $x . \alpha(x), \beta(y) \in$ $C^{1}\left(R_{+}, R_{+}\right)$are nondecreasing with $\alpha(x) \leq x, \beta(y) \leq y . \psi, \omega \in C\left(R_{+}, R_{+}\right)$are nondecreasing with $\psi(x)>0, \omega(x)>0$ for $x \in(0, \infty)$ and $\int_{t_{0}}^{\infty} \frac{1}{\psi\left(\phi^{-1}(s)\right)} d s=\infty$, where $0<t_{0}<a(0,0)$.

If $u \in C\left(R_{+} \times R_{+}, R_{+}\right)$satisfies the following integral inequality containing multiple integrals

$$
\begin{aligned}
\phi(u(x, y)) & \leq a(x, y)+\int_{0}^{\beta(y)} \int_{0}^{\alpha(x)}\left[f_{1}(s, t) \psi(u(s, t))+g_{1}(s, t) \psi(u(s, t)) \omega(u(s, t))\right. \\
& \left.+\int_{0}^{t} \int_{0}^{s} h_{1}(\xi, \tau) \psi(u(\xi, \tau)) d \xi d \tau\right] d s d t \\
& +\int_{0}^{y} \int_{0}^{x}\left[f_{2}(s, t) \psi(u(s, t))+g_{2}(s, t) \psi(u(s, t)) \omega(u(s, t))\right. \\
& \left.+\int_{0}^{t} \int_{0}^{s} h_{2}(\xi, \tau) \psi(u(\xi, \tau)) d \xi d \tau\right] d s d t
\end{aligned}
$$

then we can find some $\bar{x}>0, \bar{y}>0$ so that for all $x \in[0, \bar{x}], y \in[0, \bar{y}]$

$$
Y(\tilde{H}(x, y))+\int_{0}^{\beta(y)} \int_{0}^{\alpha(x)} g_{1}(s, t) d s d t+\int_{0}^{y} \int_{0}^{x} g_{2}(s, t) d s d t \in \operatorname{Dom}\left(Y^{-1}\right)
$$

and

$$
\begin{aligned}
u(x, y) & \leq \phi^{-1}\left\{J ^ { - 1 } \left[Y ^ { - 1 } \left(Y(\tilde{H}(x, y))+\int_{0}^{\beta(\gamma)} \int_{0}^{\alpha(x)} g_{1}(s, t) d s d t\right.\right.\right. \\
& \left.\left.\left.+\int_{0}^{\gamma} \int_{0}^{x} g_{2}(s, t) d s d t\right)\right]\right\}
\end{aligned}
$$

where $J, Y$ are defined as in Theorem 2.2, and

$$
\begin{aligned}
\tilde{H}(x, y)= & J(a(x, y))+\int_{0}^{\beta(\gamma)} \int_{0}^{\alpha(x)}\left[f_{1}(s, t)+\int_{0}^{t} \int_{0}^{s} h_{1}(\xi, \tau) d \xi d \tau\right] d s d t \\
& +\int_{0}^{y} \int_{0}^{x}\left[f_{2}(s, t)+\int_{0}^{t} \int_{0}^{s} h_{2}(\xi, \tau) d \xi d \tau\right] d s d t .
\end{aligned}
$$

Proof: The process for seeking for $\bar{x}, \bar{y}$ can also be referred to Remark 1 in [10].

If we take $x=0$ or $y=0$, then (35) holds trivially. Now fix $x_{0} \in(0, \bar{x}], y_{0} \in(0, \bar{y}]$, and $x \in\left(0, x_{0}\right], y \in\left(0, y_{0}\right]$. Let

$$
\begin{aligned}
z(x, y)= & a\left(x, y_{0}\right)+\int_{0}^{\beta(\gamma)} \int_{0}^{\alpha(x)}\left[f_{1}(s, t) \psi(u(s, t))+g_{1}(s, t) \psi(u(s, t)) \omega(u(s, t))\right. \\
& \left.+\int_{0}^{t} \int_{0}^{s} h_{1}(\xi, \tau) \psi(u(\xi, \tau)) d \xi d \tau\right] d s d t \\
& +\int_{0}^{\gamma} \int_{0}^{x}\left[f_{2}(s, t) \psi(u(s, t))+g_{2}(s, t) \psi(u(s, t)) \omega(u(s, t))\right. \\
& \left.+\int_{0}^{t} \int_{0}^{s} h_{2}(\xi, \tau) \psi(u(\xi, \tau)) d \xi d \tau\right] d s d t .
\end{aligned}
$$

Considering $a(x, y)$ is nondecreasing, we have $u(x, y) \leq \varphi^{-1}(z(x, y)) \leq \varphi^{-1}\left(z\left(x_{0}, y\right)\right)$. Moreover, 


$$
\begin{aligned}
\leq\left\{\beta^{\prime}(y)\right. & \int_{0}^{\alpha\left(x_{0}\right)}\left[f_{1}(s, t)+g_{1}(s, \beta(\gamma)) \omega(u(s, \beta(\gamma)))+\int_{0}^{\beta(\gamma)} \int_{0}^{s} h_{1}(\xi, \tau) d \xi d \tau\right] d s \\
& +\int_{0}^{x_{0}}\left[f_{2}(s, \gamma) \psi(u(s, y))+g_{2}(s, y) \omega(u(s, y))\right. \\
& \left.\left.+\int_{0}^{y} \int_{0}^{s} h_{2}(\xi, \tau) d \xi d \tau\right] d s\right\} \psi\left(\phi^{-1}\left(z\left(x_{0}, \gamma\right)\right)\right) .
\end{aligned}
$$

So

$$
\begin{aligned}
& \frac{z_{\gamma}\left(x_{0}, \gamma\right)}{\psi\left(\phi^{-1}\left(z\left(x_{0}, \gamma\right)\right)\right)} \leq \beta^{\prime}(\gamma) \int_{0}^{\alpha\left(x_{0}\right)}\left[f_{1}(s, \beta(\gamma))+g_{1}(s, \beta(\gamma)) \omega\left(\phi^{-1}(z(s, \beta(\gamma)))\right)\right. \\
& \left.\quad+\int_{0}^{\beta(\gamma)} \int_{0}^{s} h_{1}(\xi, \tau) d \xi d \tau\right] d s+\int_{0}^{x_{0}}\left[f_{2}(s, \gamma) \psi(u(s, \gamma))+g_{2}(s, \gamma) \omega\left(\phi^{-1}(z(s, y))\right)\right. \\
& \left.\quad+\int_{0}^{y} \int_{0}^{s} h_{2}(\xi, \tau) d \xi d \tau\right] d s .
\end{aligned}
$$

Integrating (38) from 0 to $y$ we have

$$
\begin{aligned}
& J\left(z\left(x_{0}, y\right)\right)-J\left(a\left(x_{0}, y_{0}\right)\right) \leq \\
& \quad \int_{0}^{\beta(y)} \int_{0}^{\alpha\left(x_{0}\right)}\left[f_{1}(s, t)+g_{1}(s, t) \omega\left(\phi^{-1}(z(s, t))\right)+\int_{0}^{t} \int_{0}^{s} h_{1}(\xi, \tau) d \xi d \tau\right] d s d t \\
& \quad+\int_{0}^{y} \int_{0}^{x_{0}}\left[f_{2}(s, t)+g_{2}(s, t) \omega\left(\phi^{-1}(z(s, t))\right)+\int_{0}^{t} \int_{0}^{s} h_{2}(\xi, \tau) d \xi d \tau\right] d s d t .
\end{aligned}
$$

Let

$$
\begin{aligned}
u\left(x_{0}, \gamma\right) & =J\left(a\left(x_{0}, y_{0}\right)\right)+\int_{0}^{\beta(\gamma)} \int_{0}^{\alpha\left(x_{0}\right)}\left[f_{1}(s, t)+g_{1}(s, t) \omega\left(\phi^{-1}(z(s, t))\right)\right. \\
& \left.+\int_{0}^{t} \int_{0}^{s} h_{1}(\xi, \tau) d \xi d \tau\right] d s d t+\int_{0}^{y} \int_{0}^{x_{0}}\left[f_{2}(s, t)+g_{2}(s, t) \omega\left(\phi^{-1}(z(s, t))\right)\right. \\
& \left.+\int_{0}^{t} \int_{0}^{s} h_{2}(\xi, \tau) d \xi d \tau\right] d s d t .
\end{aligned}
$$

Then

$$
\begin{aligned}
z\left(x_{0}, y\right) \leq & J^{-1}\left[u\left(x_{0}, \gamma\right)\right] \\
\leq & J^{-1}\left[\tilde{H}\left(x_{0}, \gamma_{0}\right)+\int_{0}^{\beta(\gamma)} \int_{0}^{\alpha\left(x_{0}\right)} g_{1}(s, t) \omega\left(\phi^{-1}(z(s, t))\right) d s d t\right. \\
& \left.+\int_{0}^{y} \int_{0}^{x_{0}} g_{2}(s, t) \omega\left(\phi^{-1}(z(s, t))\right) d s d t\right] .
\end{aligned}
$$

Furthermore let

$$
\begin{aligned}
v\left(x_{0}, y\right)= & \tilde{H}\left(x_{0}, y_{0}\right)+\int_{0}^{\beta(y)} \int_{0}^{\alpha\left(x_{0}\right)} g_{1}(s, t) \omega\left(\phi^{-1}(z(s, t))\right) d s d t \\
& +\int_{0}^{y} \int_{0}^{x_{0}} g_{2}(s, t) \omega\left(\phi^{-1}(z(s, t))\right) d s d t .
\end{aligned}
$$


Then

$$
z\left(x_{0}, y\right) \leq J^{-1}\left[v\left(x_{0}, y\right)\right]
$$

and

$$
\begin{aligned}
v_{y}\left(x_{0}, y\right)= & \beta^{\prime}(\gamma) \int_{0}^{\alpha\left(x_{0}\right)} g_{1}(s, \beta(\gamma)) \omega\left(\phi^{-1}(z(s, \beta(\gamma)))\right) d s \\
& +\int_{0}^{x_{0}} g_{2}(s, y) \omega\left(\phi^{-1}(z(s, y))\right) d s \\
& \leq\left[\beta^{\prime}(\gamma) \int_{0}^{\alpha\left(x_{0}\right)} g_{1}(s, \beta(\gamma)) d s+\int_{0}^{x_{0}} g_{2}(s, y) d s\right] \omega\left(\phi^{-1}\left(J^{-1}\left(v\left(x_{0}, \gamma\right)\right)\right)\right),
\end{aligned}
$$

that is,

$$
\frac{v_{\gamma}\left(x_{0}, \gamma\right)}{\omega\left(\phi^{-1}\left(J^{-1}\left(v\left(x_{0}, \gamma\right)\right)\right)\right)} \leq \beta^{\prime}(\gamma) \int_{0}^{\alpha\left(x_{0}\right)} g_{1}(s, \beta(\gamma)) d s+\int_{0}^{x_{0}} g_{2}(s, \gamma) d s .
$$

Integrating (42) from 0 to $y$, considering $v\left(x_{0}, 0\right)=\widetilde{H}\left(x_{0}, y_{0}\right)$ we have

$$
Y\left(v\left(x_{0}, y\right)\right)-Y\left(\tilde{H}\left(x_{0}, y_{0}\right)\right) \leq \int_{0}^{\beta(\gamma)} \int_{0}^{\alpha\left(x_{0}\right)} g_{1}(s, t) d s d t+\int_{0}^{\gamma} \int_{0}^{x_{0}} g_{2}(s, t) d s d t . e^{i \theta}
$$

Then

$$
v\left(x_{0}, y\right) \leq Y^{-1}\left[Y\left(\tilde{H}\left(x_{0}, y_{0}\right)\right)+\int_{0}^{\beta(y)} \int_{0}^{\alpha\left(x_{0}\right)} g_{1}(s, t) d s d t+\int_{0}^{\gamma} \int_{0}^{x_{0}} g_{2}(s, t) d s d t\right]
$$

and

$$
\begin{aligned}
u(x, y) & \leq \phi^{-1}\left[J^{-1}\left(v\left(x_{0}, y\right)\right)\right] \leq \phi^{-1}\left\{J ^ { - 1 } \left[Y ^ { - 1 } \left(Y\left(\tilde{H}\left(x_{0}, y_{0}\right)\right)\right.\right.\right. \\
& \left.\left.\left.+\int_{0}^{\beta(\gamma)} \int_{0}^{\alpha\left(x_{0}\right)} g_{1}(s, t) d s d t+\int_{0}^{\gamma} \int_{0}^{x_{0}} g_{2}(s, t) d s d t\right)\right]\right\}
\end{aligned}
$$

Take $x=x_{0}, y=y_{0}$ and we have

$$
\begin{aligned}
u\left(x_{0}, y_{0}\right) \leq & \phi^{-1}\left\{J ^ { - 1 } \left[Y ^ { - 1 } \left(Y\left(\tilde{H}\left(x_{0}, y_{0}\right)\right)+\int_{0}^{\beta\left(y_{0}\right)} \int_{0}^{\alpha\left(x_{0}\right)} g_{1}(s, t) d s d t\right.\right.\right. \\
& \left.\left.\left.+\int_{0}^{\gamma_{0}} \int_{0}^{x_{0}} g_{2}(s, t) d s d t\right)\right]\right\} .
\end{aligned}
$$

Since $x_{0} \in(0, \bar{x}], y_{0} \in(0, \bar{y}]$ are arbitrary, substitute $x_{0}, y_{0}$ with $x, y$ and the proof is complete.

Corollary 2.4: Assume that $f(x, y), g(x, y), h(x, y) \in C\left(R_{+} \times R_{+}, R_{+}\right)$, and $a, \varphi, \psi, \omega, \alpha$, $\beta, J, Y$ are defined as in Theorem 2.3. If $u \in C\left(R_{+} \times R_{+}, R_{+}\right)$satisfies the following integral inequality containing multiple integrals

$$
\begin{aligned}
\phi(u(x, y)) \leq & a(x, y)+\int_{0}^{\beta(y)} \int_{0}^{\alpha(x)}[f(s, t) \psi(u(s, t))+g(s, t) \psi(u(s, t)) \omega(u(s, t)) \\
& \left.+\int_{0}^{t} \int_{0}^{s} h(\xi, \tau) \psi(u(\xi, \tau)) d \xi d \tau\right] d s d t
\end{aligned}
$$

then we can find some $\bar{x}>0, \bar{y}>0$ such that for all $x \in[0, \bar{x}], y \in[0, \bar{y}]$

$$
Y(\tilde{H}(x, y))+\int_{0}^{\beta(y)} \int_{0}^{\alpha(x)} g_{1}(s, t) d s d t \in \operatorname{Dom}\left(Y^{-1}\right),
$$


and

$$
u(x, y) \leq \phi^{-1}\left\{J^{-1}\left[Y^{-1}\left(Y(\tilde{H}(x, y))+\int_{0}^{\beta(y)} \int_{0}^{\alpha(x)} g(s, t) d s d t\right)\right]\right\},
$$

where

$$
\left.\widetilde{H}(x, y)=J(a(x, y))+\int_{0}^{\beta(y)} \int_{0}^{\alpha(x)}\left[f(s, t)+\int_{0}^{t} \int_{0}^{s} h(\xi, \tau) d \xi d \tau\right] d s d t\right] .
$$

Remark 5: If we take $h(x, y) \equiv 0, \psi(u(x, y))=u^{n}(x, y), \varphi(x, y)=u^{m}(x, y), m>n>0$, then Corollary 2.4 reduces to Theorem D [11, Theorem 2.1].

Corollary 2.5: Assume that $f_{i}, g_{i}(x, y) \in C\left(R_{+} \times R_{+}, R_{+}\right), i=1,2$, and $a, \varphi, \psi, \omega, J, Y$ are defined as in Theorem 2.3. If $u \in C\left(R_{+} \times R_{+}, R_{+}\right)$satisfies the following integral inequality containing multiple integrals

$$
\begin{aligned}
& \phi(u(x, y)) \leq a(x, y)+\int_{0}^{\beta(\gamma)} \int_{0}^{\alpha(x)}\left[f_{1}(s, t) \psi(u(s, t))+g_{1}(s, t) \psi(u(s, t)) \omega(u(s, t))\right] d s d t \\
+ & \int_{0}^{y} \int_{0}^{x}\left[f_{2}(s, t) \psi(u(s, t))+g_{2}(s, t) \psi(u(s, t)) \omega(u(s, t))\right] d s d t,
\end{aligned}
$$

then we can find some $\bar{x}>0, \bar{y}>0$ such that for all $x \in[0, \bar{x}], y \in[0, \bar{y}]$

$$
Y(\tilde{H}(x, y))+\int_{0}^{\beta(y)} \int_{0}^{\alpha(x)} g_{1}(s, t) d s d t+\int_{0}^{y} \int_{0}^{x} g_{2}(s, t) d s d t \in \operatorname{Dom}\left(Y^{-1}\right)
$$

and

$$
\begin{aligned}
u(x, y) \leq & \phi^{-1}\left\{J ^ { - 1 } \left[Y ^ { - 1 } \left(Y(\tilde{H}(x, y))+\int_{0}^{\beta(y)} \int_{0}^{\alpha(x)} g_{1}(s, t) d s d t\right.\right.\right. \\
& \left.\left.\left.+\int_{0}^{\gamma} \int_{0}^{x} g_{2}(s, t) d s d t\right)\right]\right\}
\end{aligned}
$$

where

$$
\tilde{H}(x, y)=J(a(x, y))+\int_{0}^{\beta(y)} \int_{0}^{\alpha(x)} f_{1}(s, t) d s d t+\int_{0}^{y} \int_{0}^{x} f_{2}(s, t) d s d t .
$$

Remark 6: If we take $f_{1}(x, y)=f_{2}(x, y) \equiv 0, \psi(u(x, y))=u^{n}(x, y), \varphi(x, y)=u^{m}(x, y), m$ $>n$, then Corollary 8 reduces to Theorem E [11, Theorem 2.2].

\section{Applications}

In this section, we will present two examples in order to illustrate the validity of the above results. In the first example, we will try to prove the global existence of the solutions of a delay differential equation, while in the second example, we will obtain the bound of the solutions of an integral equation.

For the sake of proving the global existence of solutions of differential equations, we first recall some basic facts. Consider the following equation

$$
\left\{\begin{array}{l}
X^{\prime}(t)=H(t, X(t), X(\alpha(t))) \\
X(0)=X_{0}
\end{array}\right.
$$

with $X_{0} \in R^{n}, H \in C\left(R_{+} \times R^{2 n}, R^{n}\right), \alpha \in C^{1}\left(R_{+}, R_{+}\right)$satisfying $\alpha(t) \leq t$ for $t \geq 0$. A result in [13] guarantees that for every $X_{0} \in R^{n}$, Equation 45 has a solution, but the 
uniqueness of solutions cannot be guaranteed. Furthermore, every solution of (45) has a maximal time of existence $T>0$, and if $T<\infty$, then $\lim _{t \rightarrow \infty} \sup \|X(t)\|=\infty$.

Example 1: Consider the following differential equation group

$$
\left\{\begin{array}{c}
\left(x^{p}(t)\right)^{\prime}=y^{\frac{p+q}{2}}(t)+F(x(t), t) \\
\left(y^{p}(t)\right)^{\prime}=G(t, x(\alpha(t)))
\end{array}\right.
$$

where $p$ is an even number. $\alpha(t)$ is a nondecreasing function, $\alpha(t) \in C^{1}\left(R_{+}, R_{+}\right), \alpha(t)$ $\leq t, \quad \forall t \geq 0 . \quad p>q>0$. Assume $|F(x(t), t)| \leq \tilde{f}_{1}(t)\left|x^{q}(t)\right| v(|x|)$, $\tilde{f}_{3}(t)=\max \left(1, \tilde{f}_{1}(t)\right), \tilde{f}_{3}(t)=\max \left(1, \tilde{f}_{1}(t)\right)$, where $\tilde{f}_{1}, \tilde{f}_{2}, v \in C\left(R_{+}, R_{+}\right), \int_{0}^{\infty} \frac{1}{v(s)} d s=\infty$, and $v$ is nondecreasing.

Let $u^{p}(t)=x^{p}(t)+y^{p}(t)$, then

$$
\left(u^{p}(t)\right)^{\prime}=\left(x^{p}(t)\right)^{\prime}+\left(y^{p}(t)\right)^{\prime}=y^{\frac{p+q}{2}}(t)+F(x(t), t)+G(t, x(\alpha(t)))
$$

If $(x(t), y(t))$ is a solution of (46) defined on the maximal existence interval [0,T), integrating (47) from 0 to $t$, we have

$$
\begin{aligned}
\left|u^{p}(t)\right| & \leq\left|u^{p}(0)\right|+\int_{0}^{t}\left[\left|y^{\frac{p+q}{2}}(s)\right|+|F(x(s), s)|+|G(s, x(\alpha(s)))|\right] d s \\
& \leq \int_{0}^{t}\left[\left|u \frac{p+q}{2}(s)\right|+\tilde{f}_{1}(s)\left|x^{q}(s)\right| v(|x|)+\tilde{f}_{2}(s)\left|x^{q}(\alpha(s))\right|\right] d s \\
& \leq \int_{0}^{t}\left[\left|u \frac{p+q}{2}(s)\right|+\tilde{f}(s)\left|u^{q}(s)\right| v(|u|)+\tilde{f}_{2}(s)\left|u^{q}(\alpha(s))\right|\right] d s .
\end{aligned}
$$

Then

$$
\begin{aligned}
\left|u^{p}(t)\right| & \leq\left|u^{p}(0)\right|+\int_{0}^{t}\left[\left|u \frac{p+q}{2}(s)\right|+\tilde{f}_{1}(s)\left|u^{q}(s)\right| v(|u|)+\tilde{f}_{2}(s)\left|u^{q}(\alpha(s))\right|\right] d s \\
& \leq\left|u^{p}(0)\right|+\int_{0}^{t}\left[\tilde{f}_{3}(s)\left|u^{q}(s)\right| \omega(|u|)+\tilde{f}_{2}(s)\left|u^{q}(\alpha(s))\right|\right] d s \\
& =\left|u^{p}(0)\right|+\int_{0}^{t} \widetilde{f}_{3}(s)\left|u^{q}(s)\right| \omega(|u|)+\int_{0}^{\alpha(t)} \frac{\widetilde{f}_{2}\left(\alpha^{-1} r\right)}{\alpha^{\prime}\left(\alpha^{-1} r\right)}\left|u^{q}(r)\right| d r,
\end{aligned}
$$

where $\omega\left(|u|=v(|u|)+\mid u \frac{p-q}{2}\right.$. From Theorem 2.1 we have

$$
\begin{aligned}
& |u(t)| \leq\left\{\Omega^{-1}\left[\Omega\left(\left|u^{p-q}(0)\right|+\frac{p-q}{p} \int_{0}^{\alpha(t)} \frac{\tilde{f}_{2}\left(\alpha^{-1} r\right)}{\alpha^{\prime}\left(\alpha^{-1} r\right)} d r\right)+\frac{p-q}{p} \int_{0}^{t} \tilde{f}_{3}(s) d s\right]\right\} \overline{p-q} \\
& =\left\{\Omega^{-1}\left[\Omega\left(\left|u^{p-q}(0)\right|+\frac{p-q}{p} \int_{0}^{t} \tilde{f}_{2}(s) d s\right)+\frac{p-q}{p} \int_{0}^{t} \tilde{f}_{3}(s) d s\right]\right\} \overline{p-q}, 0 \leq t<T .
\end{aligned}
$$

Obviously we have $\{|x(t)|,|y(t)|\} \leq|u(t)|$. So $x(t), y(t)$ do not blow up in finite time. Then $T=\infty$, and the solutions of (46) are global. 
Example 2: Considering the following integral equation

$$
u(x, y) \ln (u(x, y)+1)=a(x, y)+\int_{0}^{\beta(y)} \int_{0}^{\alpha(x)}[F(s, t, u(x, y))+G(s, t, u(x, y))] d s d t,
$$

where $u \in C\left(R_{+} \times R_{+}, R_{+}\right),|F(x, y, u(x, y))| \leq f(x, y) u(x, y)$,

$|G(x, y, u(x, y))| \leq g(x, y) u^{2}(x, y), f, g \in C\left(R_{+} \times R_{+}, R_{+}\right), a(x, y), \alpha(x), \beta(y)$ are defined as in Theorem 2.3.

Let $\varphi(u)=u \ln (u+1), \omega(u)=u, \eta(u)=u$. Then one can easily see the conditions of Theorem 2.3 are satisfied. So we can obtain the bound of $u(x, y)$ as

$$
u(x, y) \leq \phi^{-1}\left\{J^{-1}\left[Y^{-1}\left(Y(\tilde{H}(x, y))+\int_{0}^{\beta(y)} \int_{0}^{\alpha(x)} g(s, t) d s d t\right]\right\}, \quad x \in[0, \bar{x}], \quad y \in[0, \bar{y}],\right.
$$

where $\bar{x}, \bar{y}$ are determined similar to the process in Theorem 2.3, and

$$
\tilde{H}(x, y)=J(a(x, y))+\int_{0}^{\beta(y)} \int_{0}^{\alpha(x)} f(s, t) d s d t .
$$

Remark 7: we note that the methods in [1-12] are not available for the estimate of bound for the solution of Equation 48 .

\section{Acknowledgements}

The authors thank the referees very much for their valuable suggestions on this paper.

\section{Author details}

${ }^{1}$ School of Science, Shandong University of Technology, Zibo, Shandong 255049, China ${ }^{2}$ School of Mathematical Sciences, Qufu Normal University, Qufu, Shandong 273165, China

\section{Authors' contributions}

BZ carried out the main part of this article. Both of the two authors read and approved the final manuscript.

\section{Competing interests}

The authors declare that they have no competing interests.

Received: 9 February 2011 Accepted: 20 July 2011 Published: 20 July 2011

\section{References}

1. Li, WN, Han, MA, Meng, FW: Some new delay integral inequalities and their applications. J Comput Appl Math. 180, 191-200 (2005). doi:10.1016/j.cam.2004.10.011

2. Lipovan, O: A retarded integral inequality and its applications. J Math Anal Appl. 285, 436-443 (2003). doi:10.1016/ S0022-247X(03)00409-8

3. $\mathrm{Ma}, \mathrm{QH}$, Yang, EH: Some new Gronwall-Bellman-Bihari type integral inequalities with delay. Period Math Hungar. 44(2), 225-238 (2002). doi:10.1023/A:1019600715281

4. Yuan, ZL, Yuan, XW, Meng, FW: Some new delay integral inequalities and their applications. Appl Math Comput. 208, 231-237 (2009). doi:10.1016/j.amc.2008.11.043

5. Lipovan, O: Integral inequalities for retarded Volterra equations. J Math Anal Appl. 322, 349-358 (2006). doi:10.1016/j. jmaa.2005.08.097

6. Pachpatte, BG: Explicit bounds on certain integral inequalities. J Math Anal Appl. 267, 48-61 (2002). doi:10.1006/ jmaa.2001.7743

7. Pachpatte, BG: A note on certain integral inequalities with delay. Period Math Hungar. 31, 234-299 (1995)

8. Pachpatte, BG: On some new nonlinear retarded integral inequalities. J Inequal Pure Appl Math 5, 1-8 (2004). (Article 80)

9. Sun, YG: On retarded integral inequalities and their applications. J Math Anal Appl. 301, 265-275 (2005). doi:10.1016/j. jmaa.2004.07.020

10. Ferreira, RAC, Torres, DFM: Generalized retarded integral inequalities. Appl Math Lett. 22, 876-881 (2009). doi:10.1016/j. aml.2008.08.022

11. $\mathrm{Xu}, \mathrm{R}$, Sun, YG: On retarded integral inequalities in two independent variables and their applications. Appl Math Comput. 182, 1260-1266 (2006). doi:10.1016/j.amc.2006.05.013 
12. Li, LZ, Meng, FW, He, LL: Some generalized integral inequalities and their applications. J Math Anal Appl. 372, 339-349 (2010). doi:10.1016/j.jmaa.2010.06.042

13. Driver, R: Existence and continuous dependence of solutions of neutral functional differential equations. Arch Ration Mech Anal. 19, 149-166 (1965). doi:10.1007/BF00282279

doi:10.1186/1029-242X-2011-20

Cite this article as: Zheng and Feng: Some new nonlinear integral inequalities and their applications in the qualitative analysis of differential equations. Journal of Inequalities and Applications 2011 2011:20.

Submit your manuscript to a SpringerOpen ${ }^{\odot}$ journal and benefit from:

- Convenient online submission

- Rigorous peer review

- Immediate publication on acceptance

- Open access: articles freely available online

- High visibility within the field

- Retaining the copyright to your article

Submit your next manuscript at $\boldsymbol{s p r i n g e r o p e n . c o m ~}$ 